\title{
Archéopages
}

Archéopages

Archéologie et société

34 | 07/2012

Campagnes

\section{Arboriculture ou viticulture? Le cas de la villa gallo-romaine du Quiou}

Jean-Charles Arramond et Christophe Requi

\section{(2) OpenEdition}

1 Journals

Édition électronique

URL : https://journals.openedition.org/archeopages/375

DOI : 10.4000/archeopages.375

ISSN : 2269-9872

Éditeur

INRAP - Institut national de recherches archéologiques préventives

Édition imprimée

Date de publication : 1 février 2012

Pagination : 6-9

ISSN : 1622-8545

\section{Référence électronique}

Jean-Charles Arramond et Christophe Requi, « Arboriculture ou viticulture ? Le cas de la villa gallo-

romaine du Quiou », Archéopages [En ligne], 34 | 07/2012, mis en ligne le 01 juillet 2012, consulté le 24 janvier 2022. URL : http://journals.openedition.org/archeopages/375 ; DOI : https://doi.org/10.4000/ archeopages. 375 


\title{
Arboriculture ou viticulture? Le cas de la villa gallo-romaine du Quiou
}

\author{
Jean-Charles Arramond I \\ Christophe Requi Irrap, UMR 5008
}

1. L'intervention archéologique

programmée, en cours, débutée en 2001, est financée par l'État (ministère de la culture et de la communication SRA de l'archéologie de Bretagne), le conseil régional de Bretagne, et le conseil général des Côtes d'Armor qui s'est en outre porté acquéreur des terrains où est implantée la résidence principale.
La villa du Quiou (Côtes-d'Armor), connue dès la fin du XIX ${ }^{e}$ siècle, constitue l'un des exemplaires les plus anciens de la province de Lyonnaise occidentale $\mathbf{1}$. Elle connaît une longue durée de vie, du début du $\mathrm{I}^{\mathrm{er}}$ siècle de notre ère jusquà l'Antiquité tardive, son apogée se situant à la fin du $\mathrm{II}^{\mathrm{e}}$ siècle. L'espace continue à être occupé jusqu'au haut Moyen Âge. Cette villa présente des intérêts multiples: ses dimensions exceptionnelles, ses aménagements extérieurs et intérieurs (telles les salles de réception de l'aile nord), ou encore sa décoration peinte de grande qualité. Après une présentation générale, ce sont les plantations ( $\mathrm{I}^{\mathrm{er}}$ siècle de notre ère) qui retiendront ici notre attention. Notre connaissance ce des dernières provient de fouilles exhaustives sur $400 \mathrm{~m}^{2}$ le long de l'aile sud du bâtiment principal I et de sondages sur les parcelles situées au sud (1 ha).

\section{Composition générale de la villa}

La villa, implantée sur le tiers inférieur d'une légère pente orientée à l'ouest, présente un plan organisé autour de deux cours [ill. 1] : la première, compartimentée, intégrée au corps résidentiel à l'ouest, et la seconde, de plus grande ampleur, située dans le prolongement de celui-ci. La pars urbana (I) [ill. 2] s'articule sur les trois côtés d'une cour fermée, rectangulaire, d'environ $2000 \mathrm{~m}^{2}$; elle est complétée au nord, par un balneum (Bât. II) en saillie. Â la fin du $\mathrm{I}^{\mathrm{er}}$ siècle de notre ère, un bâtiment rectangulaire (Bât.III) est construit contre l'aile sud du corps résidentiel, élargissant ainsi la cour initiale, à l'est du portique; il précède la cour de la pars rustica. Si la morphologie de la pars urbana est connue, il n'en est pas de même des bâtiments d'exploitation, qui s'étendent vraisemblablement vers l'est autour d'une deuxième cour. Les résultats des prospections pédestre et géophysique et la découverte de trous de poteaux, de nombreuses fosses, de restes de solins et d'un foyer lors d'une opération de diagnostic mécanisée en 2007, confirment la présence, dans le secteur nord, de bâtiments appartenant à la pars rustica, qui semblent se développer jusqu'au village actuel. Sur les côtés est et sud, des concentrations de mobilier signalent également l'existence de constructions fermant la cour agricole.

\section{Disposition des fosses de plantation}

Les fosses circulaires [ill. 3] mesurent, selon leur niveau d'arasement, entre 1 et $2 \mathrm{~m}$ de diamètre pour un diamètre moyen restitué autour de $1,5 \mathrm{~m}$. Leur profondeur moyenne de o,6 $\mathrm{m}$ varie selon leur niveau d'apparition : en effet, la lecture de leur remplissage était plus aisée dans la partie nord de la zone où le sol naturel est naturellement plus haut. Dans la rangée la plus à l'ouest, les fosses sud ont été restituées car le pendage naturel du socle rocheux $(0,7 \mathrm{~m})$ n'a pas permis de discerner les remplissages des fosses avec certitude. Au niveau de la limite sud de la zone, les fosses étaient lisibles sur seulement $\mathrm{o}, 10$ à $0,20 \mathrm{~m}$ de profondeur. Dans la zone de fouille, six fosses ont été mises au jour, dans la plupart des rangées, mais la limite est inconnue. L'espacement des fosses de plantation en tout sens se situe autour de $5 \mathrm{~m}$, en moyenne, de centre à centre.

Leur creusement, en partie inscrit dans le substrat rocheux (falun), dénote la volonté de pouvoir disposer d'un volume de terre suffisant pour le développement du système racinaire (évacuation des déblais issus du socle calcaire). Les coupes stratigraphiques n'ont pas révélé d'horizon pouvant attester la présence d'une couche spécifique d'amendement. Seuls quelques charbons de bois et quelques tessons sont présents à proximité des bâtiments et absents pour les fosses les plus éloignées.

Les sondages complémentaires ont permis 


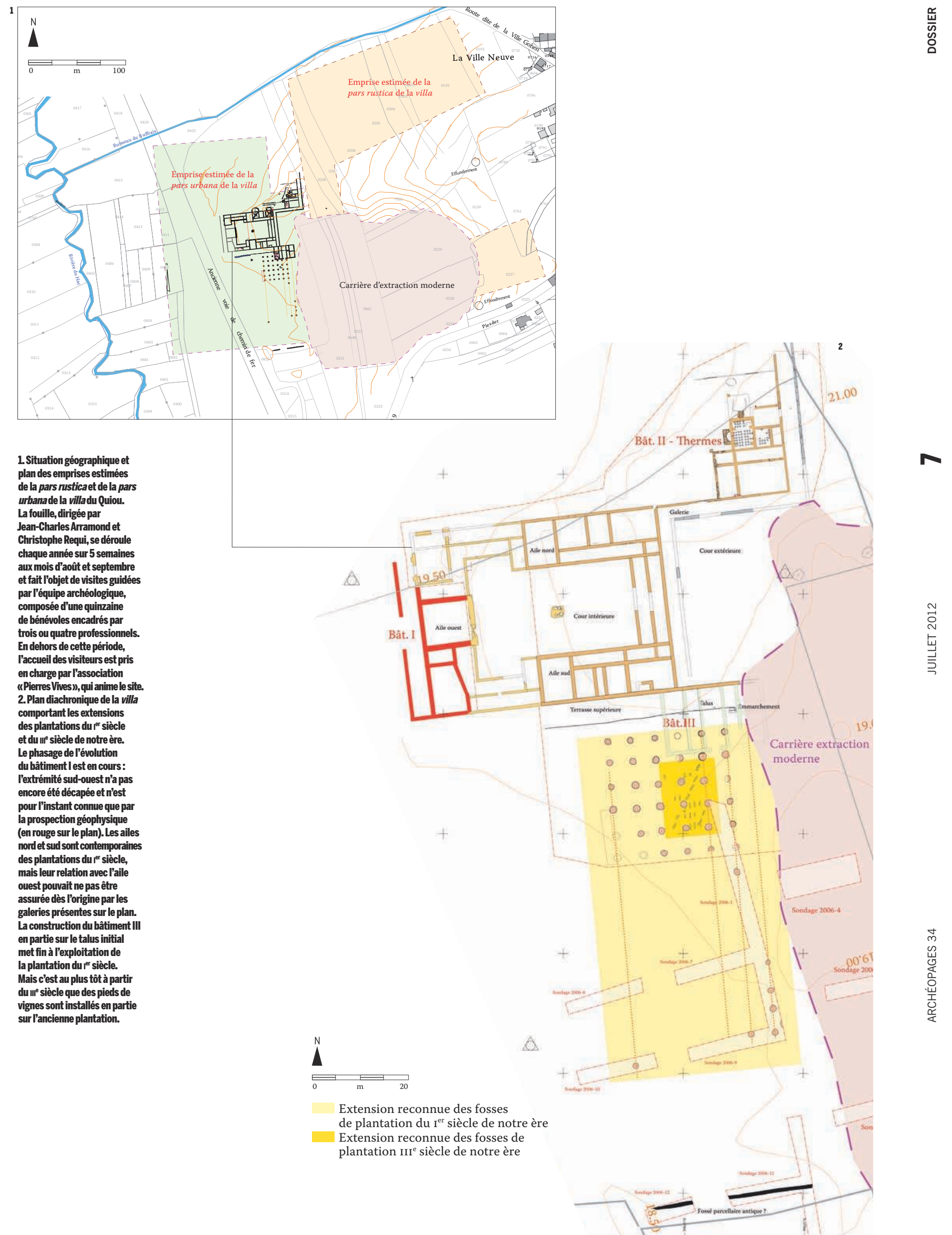



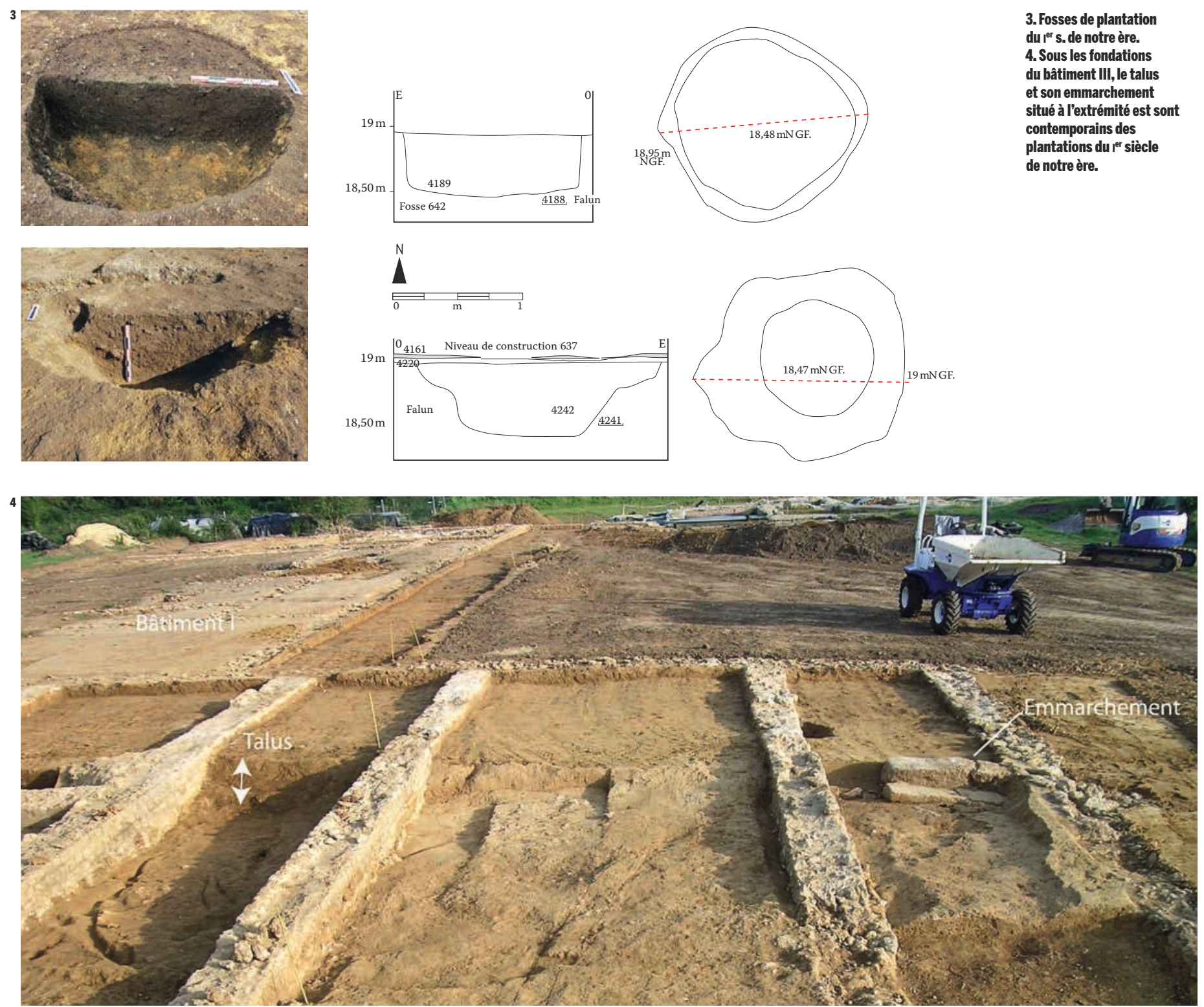

d'estimer la surface de la plantation conservée ; cependant sa limite n'est pas connue du fait d'activités d'extractions postérieures. Les fosses se développaient donc sur $2250 \mathrm{~m}^{2}$ au minimum (30 m d'est en ouest sur $75 \mathrm{~m}$ du nord au sud), au sud du bâtiment principal de la villa, en contrebas d'un talus. Sept rangées ont été identifiées avec un peu moins d'une centaine de fosses si on les restitue en continu sur les lignes. Leur disposition orthonormée cohérente avec l'orientation et l'implantation du bâtiment I de la villa permet de penser que nous sommes en présence d'une seule plantation, planifiée peut-être dès l'origine.

\section{Vocation des plantations}

La détermination des espèces plantées n'est pas possible. Il peut donc théoriquement s'agir d'une plantation de production fruitière ou d'ornement. La disposition des fosses sous la forme d'un quadrillage orthonormé et leur étendue paraît privilégier la première hypothèse qui devra être vérifiée. En tenant compte de nos connaissances actuelles et des informations fournies par les agronomes antiques, deux dévolutions paraissent envisageables : un verger ou un vignoble.

L'espacement des fosses de plantation et leur dimension sont les seuls éléments factuels dont nous disposons pour cette problématique. En effet, le niveau d'arasement des vestiges de l'aile sud de la villa n'a pas permis de mettre au jour de substructures attribuables de manière formelle à l'exploitation viticole (dolia, pressoir...). Columelle (De l'agriculture, V, IX) conseille, pour les oliviers, des fosses de 4 pieds $(1,2 \mathrm{~m})$ en tous sens, avec des espacements variables selon la nature des terrains et les éventuelles cultures intercalaires : un maillage de 60 pieds $(18 \mathrm{~m})$ sur $40(12 \mathrm{~m})$ sur «terrains gras et propre aux céréales », et un maillage de 24 pieds $(7 \mathrm{~m})$ sur «terrain maigre». Pour les fruitiers, un maillage de 30 à 40 pieds (9 à $12 \mathrm{~m}$ ) est préconisé en tout sens. Ces maillages théoriques se retrouvent au sein des exploitations antiques fouillées dans le sud de la Gaule (Jung et al., 2008) où des vergers ont été attestés par des 
espacements d'une quinzaine de mètres entre les fosses quadrangulaires. Des fosses de plantation ont néanmoins été interprétées comme des vergers avec des espacements de 3,5 à 4 m ou des plantations fruitières (baies) autour de Reims (Koehler, 2003, p.41).

$\mathrm{Si}$ les dimensions ( 3 à 4 pieds) des fosses de plantation du Quiou correspondent aux canons agronomiques antiques pour la production de fruits, les espacements se révèlent très inférieurs aux préconisations. Or ces dimensions ne peuvent être mises en relation avec une nature «maigre» des terres : en effet le bassin sédimentaire du Quiou est réputé pour la nature fertile de son terroir. La pousse des arbres plantés devait plutôt être privilégiée par la nature du sol, comme c'est toujours le cas aujourd'hui. Il peut donc s'agir de production de fruits issus de petits arbres. Mais, alors, c'est la dimension des fosses qui peut paraître disproportionnée.

\section{Vers une interprétation viticole?}

Nous proposons une autre hypothèse, étayée par un mode de culture attesté dans l'Antiquité : les vignes sur arbres ou sur hautains. La vigne pousse sur un arbre préalablement implanté et taillé de manière à constituer un support vivant «étagé » tabulatum (Billiard, 1913, p. 356-368; Columelle, V). Les espacements conseillés par Columelle pour cette méthode sont de 20 pieds $(6 \mathrm{~m})$ si l'on ne veut pas produire de céréales entre les rangs. Ce maillage est proche de celui du site du Quiou en ce qui concerne les espacements observés. Cette proposition d'interprétation va dans le sens des recherches récentes suggérant une relecture des données de fouilles anciennes qui avaient du mal à intégrer la probabilité de l'existence de vigne précoce dans le Nord-Ouest de la Gaule (Hervé-Monteil et al., 2011). L'étude des constructions de la villa démontre un respect des modes de construction et des styles décoratifs du Haut-Empire comme l'attestent d'ailleurs deux monnaies frappées sous Auguste et Tibère. Dans ce contexte, la volonté d'importer la viticulture pourrait procéder, au même titre que la planification administrative, urbaine, architecturale, de l'expansion de la culture par le nouveau pouvoir. Pour l'instant, l'absence de constructions reconnues attribuables au traitement de la vigne (pressoirs, fouloirs bétonnés...) sur la villa pourrait aller à l'encontre de cette hypothèse; cependant ces vestiges sont rares sur les sites de l'Ouest de la Gaule Lyonnaise (Hervé-Montail et al., 2011 ; Balmelle et al., 2001) où des traces de plantations ont été identifiées. La position de cette plantation à proximité $d u$ bâtiment principal I, à l'écart de la pars rustica est aussi à prendre en compte : située en contrebas d'un petit talus $(0,8 \mathrm{~m})$, la présence d'un emmarchement massif à l'extrémité est [ill. 4] permettait d'accéder à pied à la plantation sans avoir à emprunter le chemin de terre « utilitaire » qui devait y mener. Cet accès particulier permet de proposer un statut particulier à cette dernière, d'agrément ou de prestige du fait des variétés plantées. Il est donc possible d'envisager une expérimentation d'implantation de la vigne, précoce pour l'Ouest de la Gaule Lyonnaise.

La présence de la vigne sur le site est toutefois attestée plus tardivement après la construction du bâtiment III [ill. 2]. Identifiées sur une surface d'environ $100 \mathrm{~m}^{2}, 23$ fosses oblongues mesurent entre 0,7 et $0,9 \mathrm{~m}$ de long, et de 0,2 à $0,3 \mathrm{~m}$ de large pour une profondeur estimée à o,3-0,4 $\mathrm{m}$ du fait des labours. Leur forme allongée et ramassée, leurs dimensions et leur maillage correspondent aux fosses de plantation de vigne identifiées dans les provinces de Narbonnaise (Boissinot, 2001) et dans l'Ouest de la Gaule (Hervé-Monteil et al., 2011). L'observation des fosses a été perturbée par les labours et dépend de leur impact sur les couches inférieures.

Deux axes divergents se dessinent : le premier, nord-sud, surtout présent à l'emplacement d'une ancienne zone dense de gâchage, comporte des lignes ( 5 ou 6 ) distantes de $1 \mathrm{~m}$, dans lesquelles les creusements sont distants deux à deux de 0,8 à $0,9 \mathrm{~m}$; le second, nord-est/sud-ouest, comporte deux alignements parallèles (l'un de 6 fosses et le second de 2, selon les observations). Ces structures ne recoupent pas systématiquement profondément les faluns: il semble que la plupart s'arrêtent quasiment au contact de celui-ci. La limite sud n'est peut-être pas réelle en effet : à cet endroit, le niveau de gâchage qu'elles recoupent est plus profond puis s'interrompt. La nature du remplissage de ces creusements est identique au remblai encaissant. Ces creusements sont à dater au plus tôt du courant du $\mathrm{III}^{\mathrm{e}}$ siècle en fonction du phasage du site.

\footnotetext{
Références bibliographiques

Balmelle C. Brun J-P., Barraud D, Maurin L. Sillières P., Vernou Chr., 2001, « La viticulture antique en Aquitaine », Gallia, p.129-165.

Billiard R., 1913, La vigne dans l'antiquité, Lyon, $560 \mathrm{p}$

Boissinot PH., 2001, «Archéologie des vignobles antiques du sud de la Gaule», in BRUN J.P., LAubenheimer F. (dir.), La viticulture en Gaule, Gallia, 58, p. 45-68.

Boissinot PH., 2003, «Métrologie de l'arboriculture antique dans le Midi de la France», in FAvORY, F. (dir.), Métrologie agraire antique et médiévale, Actes de la table ronde d'Avignon, 8-9 décembre 1998, Besançon, Presses Universitaires Franc Comtoises, p. 37-57.

Jung C., Compan M., Figueiral I., 2008, «Les cultures et les pratiques antiques dans la cité de Béziers : l'exemple de la viticulture et de l'arboriculture sur l'autoroute A75 (Pézenas-Béziers, Hérault) ", in Viticulture et arboriculture antique sur le tracé de l'autoroute $A_{75}$ (Béziers-Pézenas). Résultats préliminaires, Girona. Consultable sur: http://hal.archives-ouvertes.fr/hal-oo598794

KOEHLER A., 2003, «Vergers antiques dans les campagnes péri-urbaines : le cas de Reims », RAP, n ${ }^{\circ}$-2, p. 37-46.

Hervé-Monteil M.-L., LuKas D., Monteil M., DieTSCH-SEllami M.-F., 2011, «Les pressoirs de Parville (Eure) et de Piriac-sur-Mer (LoireAtlantique) », La viticulture dans l'ouest de la Gaule Lyonnaise, Gallia, 68-1, p. 163-214.
} 